\title{
RACIOCÍNIO BASEADO EM CASOS
}

Reasoning Based on Cases

Un Razonamiento basado en Casos

\section{Liliane Pereira da Silva ${ }^{1}$, Sofia Mara de Souza ${ }^{2}$}

${ }^{1}$ Acadêmica do curso de Ciência da Computação, Centro Universitário UnirG, Gurupi - TO, Brasil.

${ }^{2}$ Mestre em Ciência da Computação, docente no Centro Universitário UnirG, Gurupi - TO, Brasil.

*Correspondência: R. Antônio Nunes da Silva, 2195 - Pq. das Acácias, Gurupi - TO, 77425-500

Centro Universitário UnirG - Campus I, e-mail: sbc.liliane@gmail.com

\section{Artigo recebido em 10/10/2016. Aprovado em 01/11/2016. Publicado em 04/11/2016.}

\section{RESUMO}

O artigo apresenta uma abordagem do Raciocínio Baseado em Casos, onde o mesmo nasceu a partir do modelo de solução de problemas da memória humana, inspirando assim a criação de programas para resolver problemas atuais. É uma técnica que tem por objetivo resolver um problema a partir do conhecimento de problemas passados, comparando-os com o novo problema, e adaptando assim para uma nova solução. Neste abordaremos, desde sua definição, as técnicas utilizadas, as etapas de seu desenvolvimento, bem como suas vantagens e desvantagens.

Palavras-chave: Inteligência artificial, Adaptação e Aprendizagem.

\section{ABSTRACT}

The paper presents an approach of Case Based Reasoning, where it was born from the problems of human memory solution model, thus inspiring the creation of programs to address current problems. It is a technique that aims to solve a problem from the knowledge of past problems, comparing them with the new problem, and adapting well to a new solution. This will cover, from its definition, the techniques used, the stages of its development, as well as its advantages and disadvantages.

Keywords: Artificial Intelligence, Adaptation and Learning..

\section{RESUMEN}

El artículo presenta un enfoque de Razonamiento Basado, en el que nació de los problemas del modelo de solución de la memoria humana, inspirando así la creación de programas para hacer frente a los problemas actuales. Es una técnica que tiene como objetivo resolver un problema desde el conocimiento de los problemas del pasado, comparándolos con el nuevo problema, y adaptar así a una nueva solución. Esto cubrirá, de su definición, las técnicas utilizadas, las etapas de su desarrollo, así como sus ventajas y desventajas.

Descriptores: Inteligencia Artificial, adaptación y aprendizaje.

\section{INTRODUÇÃO}

A inteligência artificial, que é uma área da Ciência da Computação, visa desenvolver mecanismos e dispositivos tecnológicos que possam simular o raciocínio humano, possui diversas técnicas, entre elas será abordado o Raciocínio Baseado em Casos (FERNANDES, 2003). 
Para Abel (2006), a procura de uma solução ou explicação para um determinado problema muitas vezes depara-se com a mesma questão em situações anteriores. Raciocínio Baseado em Casos (RBC) é um método de soluções de problemas que usa adaptações de soluções anteriores similares, adaptações de velhas soluções para encontrar novas, ou seja, raciocínios anteriores para interpretar uma nova situação ou criar

uma solução apropriada para um novo problema.

Um sistema de RBC procura soluções em uma base de casos passados que se aplicam no problema atual. Por exemplo, ao fazer uma viagem a trabalho, a pessoa escolhe um hotel baseado em experiências que teve para decidir sobre qual o melhor e não passar por problemas já vividos em outras ocasiões, ao mesmo tempo que ao planejar as atividades do trabalho que vai realizar durante sua estadia, ela vai se lembrar do que deu certo e o que deu errado anteriormente e criar novos planos.

$\mathrm{O}$ RBC resolve problemas ao recuperar e adaptar experiências passadas, chamadas casos e armazenadas em uma base de casos. Desta forma, um sistema RBC pode ser dividido em quatro elementos básicos

(WANGENHEIM ; WANGENHEIM, 2003):

- Representação do Conhecimento: em um sistema de $\mathrm{RBC}$, o conhecimento é representado principalmente em forma de casos, que descrevem experiências concretas;

- Medida de Similaridade: define como será calculada a similaridade entre a situação atual e um determinado caso a base de casos, sendo aplicada repetidamente, par a par, para todos os casos, chegando a um valor de similaridade. Estes valores e casos são ordenados e os mais similares são sugeridos como solução potencial para o problema presente;
- Adaptação: situações passadas representadas como casos dificilmente serão idênticas as do problema atual. Sistemas de RBC avançados têm mecanismos e conhecimento para adaptar os casos recuperados completamente, para verificar se satisfazem as características da situação presente;

- Aprendizado: para que um sistema se mantenha atualizado e evolua continuamente, sempre que ele resolver um problema com sucesso deverá ser capaz de lembrar-se dessa situação no futuro como mais um novo caso.

Ainda segundo os autores Wangenheim e Wangenheim, os casos são armazenados e organizados em uma base de casos, onde estão à disposição para serem reutilizados, onde este terá experiências positivas de solução que contribuíram com o sucesso para resolver um problema em específico de forma que possam ser reutilizadas. Experiências negativas, que expressam tentativas frustradas de solução também podem ser armazenadas, com o objetivo de indicar problemas potenciais e prevenir a repetição de erros passados.

Há certas observações no RBC que vem sendo exploradas como os conceitos de grau de similaridades entre processos passados e atuais e os ajustes de soluções no banco de casos para diferentes situações. Até o momento não há soluções definitivas para estes casos, embora seja explorado na literatura básica do RBC.

\section{MATERIAIS E MÉTODOS}

Na visão de Kisner (2003), o RBC pode usar várias tarefas de raciocínio como, por exemplo: propor soluções para novos problemas, antecipar, evitar e explicar fracassos em soluções propostas, e adequar ou reparar soluções propostas e assim ajustar a nova situação. Um sistema que use esta técnica pode desenvolver uma grande capacidade para 
aprender, porque seu processo de aprendizagem é simples, desde de problemas resolvidos como também podem aprender com seus fracassos.

Ao solucionar problemas no domínio, a técnica de RBC deve ser utilizada apenas de forma parcial, pois trabalha com experiências. Mas, ao armazenar um caso de sucesso ou de fracasso em sua memória o sistema, na realidade, estará aumentando a sua compreensão e o seu conhecimento do domínio. $\mathrm{E}$, isso, permite que a sua performance possa melhorar como, por exemplo:

- Aumentar a capacidade de recuperar casos mais similares aos novos problemas, o que implica em soluções mais adequadas e que exigem menos adaptações;

- Casos similares a casos passados, que foram resolvidos em vários passos devido às suas complexidades, podem agora ser solucionados mais facilmente, porque não necessitam repetir os caminhos traçados pelos outros, basta adaptar as suas soluções;

- Situações similares a casos passados que fracassaram evitam repetir o mesmo erro e transforma em indexadas por características que impeçam esses casos de serem recuperados.

O Raciocínio Baseado em Casos permite a construção de protótipo nas fases iniciais do projeto, antes da compreensão e estruturação completa do domínio, com aquisição de casos em domínios pouco estruturados ou com base teórica mal definida. Possui uma ótima amostragem dos tipos de problemas que o sistema deve resolver, além de casos possam ser bons indicadores de quais partes do problema são importantes e quais as características que o especialista considera relevantes para a solução (KISNER, 2003).
Atualmente existem diversas ferramentas voltadas para a criação das práticas de Raciocínio Baseado em Casos, as mais utilizadas são:

- CBRShell (CBR SHELL, 2009): Desenvolvido pelo grupo Artificial Intelligence Applications Institute School of Informatics da Universidade de Edinburgh. Estabelece as definições através de uma interface gráfica, porém não apresenta uma sequência lógica de passos para uso;

- MyCBR (MyCBR, 2009): Desenvolvida pelo Centro de Pesquisa Alemã de Inteligência Artificial (DFKI). O foco principal deste é minimizar o esforço para construir aplicações RBC que requerem alta intensidade cognitiva nas medidas de similaridade, tendo como principal deficiência constatada o uso apenas de arquivos XML para armazenar informações.

- JColibri (GAIA, 2009): é um framework orientado a objetos que facilita a construção de sistemas RBC desenvolvido pelo GAIA (Group for Artificial Intelligence Applications). Sua primeira versão disponibiliza um ambiente não muito usual para elaboração de sistema e sua segunda versão não possui interface gráfica para elaboração e testes do sistema RBC.

Para gerar um modelo de ferramenta RBC com uma análise de todas as características, funcionalidades e seus problemas de usabilidade, com a verificação das limitações do projeto que deseja ser criado e implementado. Um método muito utilizado de trabalho é a técnica de aprendizado por incremento. A metodologia, segundo Lakatos (2008), é o caminho determinado pelas decisões como condição necessária, mas não suficiente para atingir a verdade.

\section{RESULTADOS E DISCUSSÃO}


A ferramenta arquitetada, cujo instrumento composto é um ambiente onde usuários podem compreender o funcionamento da técnica de RBC e elaborar a mesma por meio de tutoriais, passo a passo até o objetivo final. A mesma pode ser desenvolvida diante de todos os levantamentos de requisitos, segundo os autores Wangenheim e Wangenheim (2003), são necessários como elementos básicos:

- Estrutura: É onde deve-se determinar a estrutura do caso em si, desde de seus atributos à descrição do problema, solução e seu respectivo resultado;

- Base de dados: Definição um tipo de método em específico para a base de dados, onde os casos serão recuperados e implementam um filtro inicial de dados caso houver necessidade;

- Fontes de dados: Definir uma estrutura para guardar as informações na base de casos, esta etapa também deve definir um banco de dados;

- Adaptação: Definir em quais métodos haverá ajustes ou adaptações das informações ao realizar o ciclo RBC;

- Métricas de similaridades: Tem se dois tipos: local (levando em consideração apenas um atributo de caso por vez) e global (considera todos atributos). A ferramenta oferece métricas para cada tipo de similaridades, que se encaixa de acordo com seus atributos;

- Execução de todo o ciclo: É onde o ciclo é executado e podemos entender o seu funcionamento e verificamos todos os seus resultados.

As figuras 1 e 2 ilustram modelos de como seria o projeto, onde a primeira tela mostra como é o funcionamento da ferramenta, como pode ser projetada e mostra o quanto é dinâmica e de fácil acesso. Deve ser criado o projeto no formato XML, o único formato até o momento aceito para este padrão e é usada a técnica por incremento, desde que uma experiência nova é armazenada, cada vez que o problema for resolvido, tudo permanecerá disponível imediatamente para soluções de problemas futuros.

Figura 1- JColibri. Fonte: JColibri (2016)

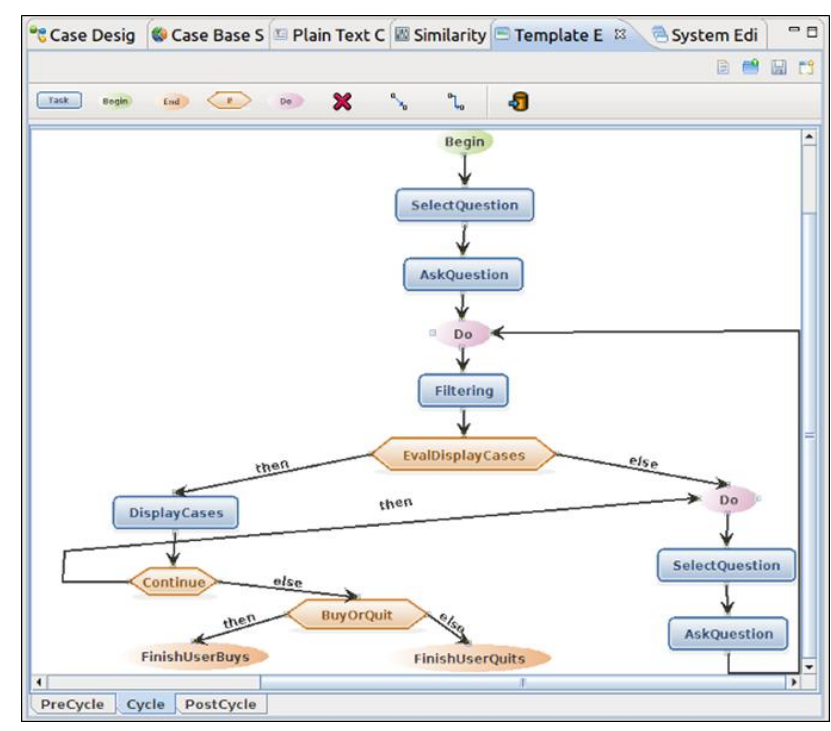

Figura 2 - Ciclo do RBC. Fonte: Wangenheim (2003)

\section{Ciclo de Vida}

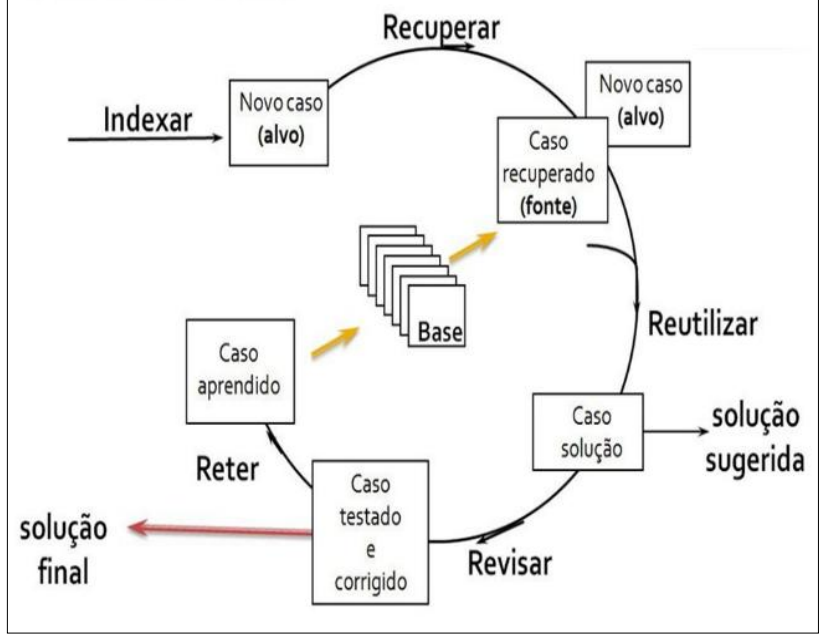

Conforme Abel (2006), as etapas que formam o ciclo para o desenvolvimento de um sistema de RBC são as representações dos casos, através da recuperação, adaptação e aprendizagem. Quando iniciada, respeitando todas as técnicas, regras $\mathrm{e}$ métodos do ciclo, representa o sucesso ou fracasso de 
um RBC. O sistema terá etapas que englobam o ciclo de casos desde o processo de recuperação, na qual faz uma busca na memória, sua reutilização, cuja característica é adaptação da solução armazenada, exigirá revisão pois desta podemos aprender com suas falhas, a retenção seleciona o que seria relevante dentro do ciclo.

A representação dos casos poderá ser realizada através de várias linguagens, seja de vetores de características, de atributos de valores como frames, objetos entre outras redes semânticas, além da lógica de primeira ordem, também podemos representar através da natureza do que se deseja representar ou verificar o problema da expressividade $\mathrm{x}$ eficiência.

Os casos de problemas recuperados são avaliados para depois serem utilizados ou armazenados na base e iniciam-se com toda a descrição da problemática em questão e finaliza com a recuperação de casos similares. A busca é através de algoritmos que seleciona o caso com as regras préestabelecidas de acordo com as suas similaridades.

As etapas de recuperação são divididas: Matching, que buscam encontrar os diversos casos mais similares ao caso alvo, e no Ranking, visa entre os casos, escolher o melhor em relação ao alvo do caso. De acordo com a necessidade são divididas: pode se tratar uma forma de reconhecer características de um caso corresponde às de outros uma maneira de calcular o grau de similaridade; verificar os objetivos da recuperação, determinar ou armazenar a importância das características.

Ainda segundo a autora, a similaridade no RBC é dividida: semântica, que não considera fatores contextuais, mas sim atributos idênticos em duas situações; estrutural, que devem estar ligados por estruturas isomórficas; organizacional, que é exigida em casos armazenados próximos a memória de casos; e a pragmática, onde duas partes são pragmaticamente similares se tiverem funções iguais.

A parte da retenção é onde o novo caso é armazenado na memória para no futuro ser usada. Alguns pontos devem ser analisados como exemplos: a remoção ocasional de casos e ser seletivo na escolha de casos para armazenamento. Já na adaptação, os métodos e estratégias são divididos em: substituição, onde troca valores da situação real por antigos; transformação, onde uma solução antiga é adaptada e adequada para uma nova situação; e outros métodos, utilizados para mudanças estruturais que não são cabíveis a outras técnicas. Os sistemas que buscam solucionar os problemas e não os de interpretação, passam por uma fase de avaliação que é similar à de justificação, onde é realizada a verificação do que do que durante a execução de uma solução ou mesmo após seu resultado ou seu término.

De acordo com o autor Kolodner (1993), as análises do projeto aplicado são estudadas e depois realizadas no final do mês, o que não permite alterar o resultado final. Se estas fossem de forma automática ou sistematizada, as mudanças poderiam ser aplicadas no decorrer do período diariamente, para assim obter melhores resultados no final do mês. Nesta situação, a técnica de Raciocínio Baseado em Casos é uma ferramenta de análise das informações útil em qualquer campo, pois os casos abordam os procedimentos adotados para as novas realidades, transformam-se assim em ferramenta de auxílio na tomada de decisão e mantem o conhecimento atribuído registrado em suas bases.

\section{CONCLUSÃO}

A técnica de Raciocínio Baseado em Casos cresce de forma avassaladora e proporciona soluções rápidas, realiza e avalia soluções quando nenhum 
método algorítmico faz avaliação, lembra experiências passadas e nos alerta do que deu errado, além de apontar as partes significativas do problema.

A utilização dessa técnica fica limitada apenas ao acesso às bases de dados completas, corretas e confiáveis que contenham entre as informações armazenadas, a descrição completa de problemas e das soluções que foram aplicadas em algum momento, pois esta é a matéria prima inicial e básica para a construção de sistemas baseados em casos. Tornou-se uma técnica natural para o reuso do conhecimento constante em repositório de dados segundo Abel (1996). As pesquisas de melhores técnicas e algoritmos dessa área promete trazer muitas novidades para esta área.

Todos os autores declararam não haver qualquer potencial conflito de interesses referente a este artigo.

\section{REFERÊNCIAS}

ABEL, MARA, REATEGUI, E. B., \& CASTILHO, J. M. V. (1996). Using case-based reasoning in a system that supports petrographic analysis. In: Braunschweig, B., \& Bremdal, B. (Ed.), In Artificial Intelligence in the Petroleum Industry (, pp. 159172). Paris: Editions Technip.

CBR Shell. CBR Tool from AIAI. 2009. Disponível em:<www.aiai.ed.ac.uk/project/cbr/cbrtools.html>.

Acesso em: 12 de novembro de 2015.

FERNANDES, A. M. R. (2003), Inteligência Artificial: noções gerais. Ed. Visual Books, Florianopolis.

GAIA - Group of Artificial Intelligence Applications. JColibri. $2009 . \quad$ Disponível:< http://gaia.fdi.ucm.es/research/colibri/colibristudio >. Acesso em: 09 de novembro de 2015.

KISNER, GILVANI BRUNO. Sistema de auxílio a restaurantes para elaboração de cardápios. 2003. Trabalho de Conclusão de Curso (Graduação em Ciência da Computação) - FURB, Blumenau,2003. Disponível em: < http://dsc.inf.furb.br/arquivos/tccs/ LAKATOS, E. M.; MARCONI, M. A. Metodologia científica. 5. ed. São Paulo: Atlas, 2008. monografias/20031gilvanibrunokisnervf.pdf $>$. Acesso em: 10 de novembro de 2015.
MYCBR. Disponível em:<http://mycbr-project.net/>. Acesso em: 13 de novembro de 2015.

WANGENHEIM, C. G.; WANGENHEIM, A. Raciocínio baseado em casos. Ed. Manole, Barueri, 2003. 